seizures and influenza A was $40.4+/-0.67^{\circ} \mathrm{C}$, whereas in those without febrile seizures it was $38.7+/-1.8^{0} \mathrm{C}(\mathrm{P}=0.04)$. Significant factors for development of febrile seizures in influenza included: family history of seizure disorders $(\mathrm{P}=0.03)$; history of febrile seizure $(\mathrm{P}=0.03)$; and coexisting gastroenteritis $(\mathrm{P}=0.05)$. History of febrile seizures was an independent risk factor $(\mathrm{P}=0.015)$. When compared to children with febrile seizures but negative influenza studies, those with confirmed influenza had a significantly higher maximum body temperature, shorter duration of fever before seizure onset, and more frequent occurrence of partial seizures. Current seizure was the first seizure in $26.5 \%$ influenza cases compared to $50 \%$ with negative viral studies $(\mathrm{P}=0.04)$. Febrile seizures were complex in $13(38.2 \%)$ influenza positive vs $7(20.6 \%)$ influenza negative cases. Duration of seizure was longer in the influenza group. (Kwong KL, Lam SY, Que TL, Wong SN. Influenza A and febrile seizures in childhood. Pediatr Neurol Dec 2006;35:395-399). (Respond: Dr Kwong, Department of Paediatrics, Tuen Mun Hospital, Tsing Chung Koon Road, Hong Kong SAR, China).

COMMENT. Influenza A is a frequent cause of febrile seizures in China and Japan but not in the United States and Europe. A recent review of the role of viral infections in the etiology of febrile seizures (Millichap JG, Millichap JJ. Pediatr Neurol Sept 2006;35:165172; idem.J Infect Dis 2004;189:564-565) concluded that fever and the height of the body temperature induced by infection is the essential factor. A threshold convulsive temperature dependent on the height of the body temperature has been established in animal and clinical studies, notably in patients with HHV-6 infection, the most common viral cause in the USA. A higher body temperature is also reported in the above series of influenza-induced febrile seizures. A specific neurotropism and CNS invasive property of HHV-6 is demonstrated in $14.5 \%$ of febrile seizure cases, using CSF-PCR analysis, and a similar neurotropic factor is suggested for influenza A virus. Only $2.5 \%$ HHV-6 cases tested showed CSF pleocytosis, a finding that tends to negate an encephalitic cause. Febrile seizures with influenza A are often complex, and difficult to differentiate from encephalopathy. Systemic immune and cytokine responses to influenza infection have also been invoked as factors. The association of influenza A and febrile seizures is seasonal, related to epidemics, especially prevalent in Asia, sometimes triggered by a concomitant reactivation of HHV infection, and often complicated by encephalopathy or complex seizures. The occurrence of febrile seizures with influenza A epidemics may also be viral strain dependent. Rapid viral diagnostic testing in patients presenting with complex febrile seizures during influenza season is recommended. Effective vaccination may prevent febrile seizures due to influenza.

\title{
ENTEROVIRUS 71 BRAINSTEM ENCEPHALITIS AND COGNITIVE AND MOTOR DEFICITS
}

Follow-up studies were conducted in 63 previously healthy children with enterovirus 71 brainstem encephalitis ( 49 stage II, 7 stage IIIa, and 7 stage IIIb) at National Cheng Kung University Hospital, Tainan, Taiwan. Mean time to follow-up was $2.8+/-1.0$ years (range 1.4-4.9 years). The ratio of boys to girls was 3 to 2 . Neurologic abnormalities on admission were altered consciousness $(47.6 \%)$, cerebellar dysfunction $(17.5 \%)$, and cranial nerve palsy $(15.9 \%)$. At follow-up, 51 had no deficits, 3 had died, and $9(14.3 \%)$ had residual deficits, cognitive in 3, cerebellar in 7, and severe motor and respiratory failure in 2. (Huang M-C, 
Wang S-M, Hsu Y-W, Lin H-C, Chi C-Y, Liu C-C. Long-term cognitive and motor deficits after enterovirus 17 brainstem encephalitis in children. Pediatrics Dec 2006;118:1785-1788). (Respond: Ching-Chuan Liu MD MPH, Department of Pediatrics, National Cheng Kung University Hospital, 138 Sheng Li Rd, Tainan, 70428, Taiwan).

COMMENT. Nonpolio enterovirus infections are most common in young children, and are spread by fecal-oral and respiratory routes. Enterovirus 71 (EV71) causes hand, foot, and mouh disease or herpangina, but infection can progress to a polio-like, acute flaccid paralysis or brainstem encephalitis (AAP Red Book, $27^{\text {th }}$ ed, 2006;284-5). The rate of CNS complications in Taiwan 1998 epidemic was estimated at 3.1 per 1000 EV71 infections, brainstem encephalitis the most common. The highest mortality occurs in patients with stage IIIb and pulmonary edema. Stage II, the most frequent, an isolated brainstem encephalitis, is characterized by myoclonus, ataxia, nystagmus, oculomotor palsies, and bulbar palsy, and stage IIIa, an autonomic nervous system dysregulation, with cold sweats, tachycardia, and hypertension. Close monitoring of cerebellar dysfunction, present in $10 \%$ of stage II cases at 3 years after hospitalization, is recommended. Prompt pulmonary resuscitation is necessary in stage IIIb cases, to prevent hypoxic-ischemic encephalopathy.

\section{ATTENTION DEFICIT DISORDERS}

\section{ATOMOXETINE-INDUCED ELECTROCARDIOGRAM CHANGES}

An 11-year-old boy with attention deficit hyperactivity disorder (ADHD) who developed palpitations and unusual cardiac repolarization changes on the electrocardiogram (ECG) during standard dose treatment with atomoxetine is reported from Parkview Clinic and Birmingham Children's Hospital, Birmingham, UK. The boy had no history of cardiac or other medical problems. He was previously treated with Concerta and developed tics. Atomoxetine was substituted in a dose of $40 \mathrm{mg}(1.1 \mathrm{mg} / \mathrm{kg} / \mathrm{day})$. After $10 \mathrm{months}$ treatment, he developed sensations of fluttering in his chest and pallor, with increasing frequency, about 3 times a week, in episodes lasting about $10 \mathrm{~min}$ each. These symptoms were not associated with exertion. The ECG showed sinus rhythm, with unusual repolarization changes after the T wave, and a QTc of $0.32 \mathrm{sec}$. Both symptoms and ECG abnormalities resolved after stopping the drug. The QTc when off the atomoxetine was $0.31 \mathrm{sec}$. An echocardiogram was normal. Although ECG testing is not mandatory during atomoxetine treatment for ADHD, further research is recommended to study long-term effects of atomoxetine on the cardiovascular system. (Rajesh AS, Bates G, Wright JGC. Atomoxetine-induced electrocardiogram changes. Arch Dis Childhood Dec 2006;91:1023-1024). (Respond: Dr AS Rajesh, Parkview Clinic, 60 Queensbridge Road, Moseley, Birmingham B13 8QE, UK).

COMMENT. Atomoxetine is a nonstimulant noradrenergic drug licensed for treatment of ADHD. In initial pediatric trials of atomoxetine, cardiovascular adverse events were rare, but in adult studies, palpitation was a significant side effect $(3.7 \%$ vs $0.8 \%$; $\mathrm{p}=0.037)$. This appears to be the first report of atomoxetine-induced ECG repolarization changes with palpitations in a child. Abnormal blood pressure elevation is noted occasionally wih atomoxetine in our own clinic, and has been reported by others (Dworkin N. J Am Acad Child Adolesc Psychiatry 2005;44:510; Ped Neur Briefs 2005;19:48). 